\title{
XXXII. On the transformation of gravity
}

\author{
James Croll LL.D. F.R.S.
}

To cite this article: James Croll LL.D. F.R.S. (1876) XXXII. On the transformation of gravity , Philosophical Magazine Series 5, 2:11, 241-254, DOI: 10.1080/14786447608639103

To link to this article: http://dx.doi.org/10.1080/14786447608639103

曲 Published online: 13 May 2009.

Submit your article to this journal 준

Џ Article views: 3

Q View related articles $₫$ 
LONDON, EDINBURGH, AND DUBLIN

PHILOSOPHICAL MAGAZINE

AND

JOURNAL OF SCIENCE.

\author{
[FIFTH SERIES.] \\ $O C T O B E R 1876$.
}

XXXII. On the Transformation of Gravity. By JAMES CroLl, LL.D., F.R.S.*

$G^{R A V I T Y}$ in relation to Correlation.-Is gravity convertible into other forms of energy? Can gravity be converted into heat, electricity, magnetism, \&c.? or can those forms of energy be converted into gravity? One, I presume, may read all that has been written on correlation and conservation of energy without finding a clear, distinct, and satisfactory answer to the question. Few will admit that gravity forms an exception to the great principle of correlation, standing isolated from all other forms of energy. It may be true that gravity cannot be directly transformed into heat, electricity, magnetism, chemical affinity, \&c., nor those forms directly transformed into gravity; but nevertheless the thing may be done indirectly. It is well known that if energy (no matter under what form) can be converted into motion or mechanical work, it can then be transformed into other forms of energy. For example, if heat is made to perform mechanical work, say to drive a machine, then that machine may be made to generate electricity, and the electricity may in turn be converted into other forms of energy. Or electricity may drive the machine, then the machine may generate heat. The heat is here not directly converted into electricity, nor the electricity directly into heat; but it is done indirectly. The heat is first converted into mechanical energy and then into electricity. The electricity is first converted into mechanical energy, then into heat. Now gravitation will drive a machine

* Communicated by the Author, having been read before the British Association, September 1876.

Phil. Mag. S. 5. Vol. 2. No. 11. Oct. 1876.

$\mathrm{R}$ 
as effectually as either heat or electricity; and the energy thus employed is as really and truly converted into mechanical energy as was the heat or the electricity in the cases to which we have referred. An electrical machine driven by waterpower will as effectually produce electricity as though it were driven by steam-power or by horse-power; and the electricity produced will as truly be derived from gravity in the one case as it would be from heat or from animal. power in the other cases.

If then it be admitted, as it must be, that in the case of the electricity produced by. the machine driven by steam-power the electricity previously existed under the form of heat, for the same reason it ought to be admitted that the electricity produced by the machine when driven by water-power previously existed under the form of gravity.

We may now suppose the process reversed and electricity to be the motive power. Conceive a maehine of some kind or other driven by electricity, and this machine to be employed in the production of heat, say by friction, and to be employed also in raising water. It will be universally admitted that we have in the one case electricity converted into heat; but it is equally evident that we have in the other case electricity converted into gravity. If the electricity produced by the descent of the water be gravity transformed into electricity, then the ascent of the water produced by electricity must be electricity transformed into gravity ; for it is the same process merely reversed.

Gravity in relation to Conservation.-If gravity be correlated to other forms of energy, it must, like them, come under the great principle of conservation. But here we enter upon debatable ground. It is admitted that gravity can perform mechanical work, and the mechanical work can be converted into other forms of energy. Here we have correlations; but it is generally denied that there is a decrease or loss of gravity resulting from such transformations. But this appears to me to be a virtual denial of the principle of conservation. To suppose a steam-engine to perform the work of raising the loaded piston, without the steam sustaining any loss of heat, would be universally admitted to be a violation of the principle of conservation. Every one would maintain such a thing to be impossible, and that the steam must not simply lose heat but lose an amount equivalent to the work performed. Apply the same mode of reasoning to gravity. If gravity perform mechanical work in pulling down the piston, then there must be a loss of that form of energy equivalent to the work performed. But it is not admitted that gravity is 
diminished or is weakened by the work which it performs. If a stone one pound in weight fall from an elevation of 1000 feet, one thousand foot-pounds of work will have been performed by gravity on the stone. The stone on reaching the ground is possessed of that amount of energy in the form of motion, all of which has been derived from gravity. But gravity, it is nevertheless maintained, has sustained no loss in communicating this amount of energy to the stone.

The reasons which appear to have led to this opinion are, I think, mainly the two following:-1. It has been assumed that the weight of a body is not affected by the work which it performs. 2. The force by which bodies are drawn towards each other does not diminish as they approach, but on the contrary increases. The mutual attraction of the stone and the earth (gravity) does not diminish as the stone descends, but the reverse; for the stone on reaching the earth's surface is attracted with greater force than it was before it began to descend. From this it is naturally inferred that, whatever may have been lost in the imprarting of motion to the stone, it cannot have been gravity.

In reference to the first of these reasons, it may be remarked that although what we call the permanent weight of a body is not affected by the work performed, still it does not follow that the weight may not be affected at the time the work is being performed, and still less that there may not be a loss of gravity resulting from the performance of the work. And in regard to the second reason, it does not necessarily follow that, because the force of gravity acting on bodies increases as they approach, there is not a loss of force resulting from their approach. A person, for example, may be growing richer every day, and yet at the same time his daily expenditure may be on the increase. Two electric currents parallel and flowing in the same direction mutually attract each other; and this attractive force increases as they are drawn together; but we know that there is at the same time a loss of attractive force resulting from their approach. When the two currents are in the act of approaching towards each other, an induced current takes place in each wire in the opposite direction to the primary current, the effect of which is to weaken the primary currents, and consequently to diminish the force of their attraction; so that when the currents are approaching each other the force of their attraction at any given moment is somewhat less than it would otherwise be were the currents stationary. The same is true of magnets. When an electromagnet is drawing towards it a bar of soft iron the attractive force of the magnet for the iron increases 


\section{Dr. J. Croll on the Transformation of Grarity.}

as the bar approaches; but there is, notwithstanding, a slight loss of attractive force resulting from the approach of the bar. The attractive force of the magnet for the bar when it is in the act of approaching is less at any given distance than it would otherwise be were the bars stationary at that distance ; for the approach of the magnetized iron weakens the magnet by weakening the current surrounding it. It is also probably true, though it cannot be so easily proved, that a permanent magnet is weakened by the approach of the magnetized iron. May not the same be true of bodies approaching each other under the mutual influence of the attractive force of gravity? May not a stone when in the act of falling be acted upon by gravity with less force at any given moment than it would be were the stone at rest at that instant? The point has never yet been determined either by experiment or by observation.

The fact that the attractive force of bodies increases as they approach towards each other cannot, therefore, be regarded as ovidence that no loss of force results from their approach. It may be here also remarked that neither can the fact of this increase of attractive force as bodies approach be regarded as in any way opposed to the law of conservation; for, as was pointed out by Professor Rankine in his reply to Faraday's objection *, no law of conservation is applicable to the simple force or tendency of two bodies to approach each other,the physical reason being that the things conserved cannot be two forces; for the one is never directly convertible into the other. What must be conserved is the force, and the work which it performs. But if the force of gravity does not sustain any loss as work is performed by it, what, then, is it that is supposed to sustain the loss? Some form of energy must diminish as work is performed; and if it bo not gravity, it must be something else.

The generally received explanation is this : when a body is projected upwards, the potential form of energy into which the upward motion of the body is transformed does not consist in the simple force of gravity or tendency of the body to descend, but consists in this force or tendency multiplied by the distance through which it is capable of descending. If a stone one pound in weight is at an elevation of 100 feet, then one pound multiplied by 100 feet gives 100 foot-pounds of potential energy possessed by the stone at that elevation; and this is usually termed the energy of position. Again, when the body has descended to within, say, 20 feet of the ground, it possesses only 20 foot-pounds of potential energy in virtue of position; for one pound multiplied by 20 feet

* Phil. Mag. [IV.] vol, xvii. p. 250 (1859). 
Dr. J. Croll an the Transyumation of Gracity. $\quad 245$

gives 20 foot-pounds; and when the ground is reached the potential energy is gone ; for although the force of gravity is not diminished, yet the potential energy is consumed, there being now no space through which the force can act. Kinetic energy thus generated being measured by the mass of the stone multiplied by the half-square of its velocity, the sum of the potential and the kinetic energies must therefore always remain constant. When the stone reaches the ground it is in possession of the 100 foot-pounds of energy in the form of motion. When it was halfway down it possessed 50 footpounds in the kinetic form of motion, and another 50 footpounds in the potential form; for, having another 50 feet to fall, its potential energy was $1 \times 50=50$ foot-pounds.

This mode of viewing the matter, it is perfectly true, completely meets the mathematical and mechanical conditions of the problem; but for this very reason it seems to me to hide somewhat the real physical nature of the process.

On a former occasion* I briefly endeavoured to show that the fundamental objection to this mode of explanation is that it makes space a form of energy, whereas, when we analyze the process properly, we find that time and space can, in the very nature of things, be nothing more than conditions. The object of the present paper is to discuss more fully this objection.

Time and space are but conditions in the process of transformation.- Space and time are conditious, but conditions absolutely necessary, to the transformation of potential energy into kinetic and of kinetic energy into potential; but they themselves cannot be forms of energy. But if it be true that the mere force of gravity or tendency of the stone to fall to the ground is not the potential energy, but that this potential energy is the force $x$ the space through which it can act, then space must become a form of potential energy. This is evident ; for the potential energy in this case consists of two factors, one of which is the space through which the force acts. It thus becomes just as much a form of energy as the other factor, viz. the force.

The reason why this mode of explanation fulfils the mathematical conditions of conservation is obvious. Space, like time, being a condition absolutely essential to the transformation of potential energy into kinetic, it follows that, no matter how much potential energy may be in the raised stone, no transformation can possibly take place if the space condition is not supplied, and the amount of transformation will just be in proportion to the space supplied. The stone may be raised to

* Phil. Mag. Decemuer 1867. 


\section{Dr. J. Crall on the Transformation of Gravity.}

any height; but if it can only descend through one foot and no more, one foot-pound of the potential energy stored up in the form of gravity can be transformed into the kinetic form ; but if two feet, three foet, ten feet, fifty feet, and so on be supplied, two foot-pounds, three foot-pounds, ten foot-pounds, and so on of potential energy will be converted into kinetic. The amount of transformation will be in proportion to the amount of the space condition supplied. When the stone reaches the ground no further transformation can take place, not because the potential energy is exhausted, but because no further space condition is available. But were a hole dug through the earth the stone would continue its descent, and transformation would go on till the earth's centre was reached, when the entire amount of potential energy of gravity acting on the stone would have disappeared and no further transformation of potential energy into kinetic would be possible-not, however, as before, because there was no more space condition, but because there would be no more gravitating force acting on the stone.

It is gravity, and gravity alone, which imparts motion to the stone. No work is performed on the stone by space; space and time merely supply the conditions for the work being done. That which, in the potential state, becomes transformed into kinetic energy must be gravity, not space. The kinetic energy which appears as the stone descends must have previously existed in the form of gravity, not as space. This truth is so self-erident that it can hardly be denied by any one who will reflect on the subject. But if the kinetic energy be derived from gravity, then there must be a decrease of gravity proportionate to the increase of kinetic energy, or else the principle of conservation is violated. If a force be transformed into something else, say into kinetic energy, then it cannot be what it was before transformation, but must be what it is transformed into, viz. kinetic energy*.

* Mr. Lewes, in his 'Problems of Life and Mind' (rol. ii. p. 353), lass it down as a fundamental principle that force is invariant and cannot be expended or even transformed. Every unit of force, according to him, remains unalterably the same in amount. His views seem to be:-that when the forces balance one another there is rest, static equilibrium; but when there is an excess of pressure in one direction, motion or vis viva results; but the forces themselves are invariant, and never increase or diminish. This is just the fundamental error which I have been cambating. Force, I consider, is energy in the potential form; motion is energy in the kinetic form. Neither the one nor the other remains constant, but both are in a state of change. That which remains constant is the sum total of both. And as the one is convertible into the other, it necessarily follows that the one must decrease as the other increases, and vice tersî́. 
But it is not in reference to gravity alone that this space condition is essential to the transformation of potential into kinetic energy. It is, as we shall shortly see, a condition absolutely necessary to the transformation of energy under every possible form. In the unbending of a spring the amount of work which can be performed is proportionate to the pressure of the spring multiplied by the space through which the pressure can act. The potential element, which in this case decreases as work is performed, is the tension or elastic force of the spring. Space is necessary simply to allow this tension to expend itself in work. It is this tension, not space, which reappears as work or kinetic energy. Take also the case of the steam-engine. The amount of work which can be performed is proportionate to the pressure of the steam in the piston multiplied by the space through which the piston can act. When the piston reaches the end of the cylinder, no more work or transformation of potential energy into kinetic is possible, not because there is no more pressure or potential energy to be transformed, but because there is no more space to allow of transformation. But in order to secure an inexhaustible supply of the space condition the ingenious device of " reversing the stroke" has been adopted-that is, of transferring the pressure to the opposite side of the piston when it reaches the end of the cylinder. By this device as much space becomes available as would be were the cylinder made a thousand miles in length. In fact the space condition becomes thus illimitable. But, be it observed, although the work performed is proportionate to the pressure of the steam multiplied by the space through which the pressure acts, still it is the pressure, and the pressure alone, which performs the work. True the pressure in the cylinder may be kept constant; but this can only be done by admitting more steam from the boiler. The energy which performs the work, or, in other words, which becomes transformed into work, is altogether derived from the boiler; the reversal of the stroke simply supplies the space condition for transformation. The energy which disappears as work is performed is heat, not space. The work performed represents so much heat lost. The work is the equivalent of the lost heat, not of the space passed over. Whatever we have gained in work we have lost in heat.

Every physicist will readily admit this conclusion in reference to an engine propelled by heat, because to suppose that that could perform mechanical work without sustaining loss would in fact be a violation of the principle of conservation. But gravitation turns our water-wheels and grinds our 
corn, performing mechanical work as truly as it is done by heat; and all this is supposed to take place without gravitation sustaining any loss. The only thing supposed to be lost is position or space to pass over. Gravity is the real agent that performs the work; and yet it is supposed to sustain no loss thereby. Heat in the steam-engine is the agent that performs the work; but it would be considered absurd to say that no heat was lost, and that what was lost was simply space in the cylinder, along which the piston could move. The absurdity seems, however, to be quite as great in the one case as in the other; and it appears as much a violation of the principle of conservation to suppose that gravitation could perform work without loss as that heat could do so. That form of energy called heat is weakened by the act of raising the loaded piston against gravity. May not that form of energy called gravity be also weakened by the act of pulling the piston down?

It is true that the pressure of the steam has a dynamical origin, and that in the performance of work there is a loss of pressure resulting from a loss of molecular motion. May not the same be equally true of gravity? In fact, if gravity has a dynamical origin, it must hold equally true of gravity and of heat. If the force of gravity, for example, results (as it, however, probably does not) from the impact of ultramundane corpuscles, as advanced by M. Le Sage, then there must be a loss of force resulting from a loss of corpuscular motion when work is performed, as truly as in the case of the steam-engine; in fact the two cases are exactly parallel.

That space is not a form of energy but simply a condition, becomes further evident from the nature of kinetic energy in the form of motion. The kinetic energy of a moving body is equal to its mass multiplied by the half-square of its velocity. Now it must be observed that the energy possessed by the body depends upon its velocity, and is entirely independent of the amount of space passed over. A body moving with a certain velocity possesses the same amount of energy whatever may be the space passed over. A one-pound weight moving at the rate of 1000 feet per second possesses 15,625 foot-pounds of energy, whether it moves over one thousandth of a hairbreadth or over one thousand miles. It would not possess any more energy without a change of velocity though it were to continue its course onward in space through all eternity. The energy possessed is the same whether the space passed over be infinitely great or infinitely small, which would not be the case if space were itself a form of energy.

In order to perceive more clearly the true relationship of 
time and space to the transformation of energy, it will be necessary to treat the subject in a more general and abstract form, so as to subject the process to a eloser analysis.

Time and Space in relation to ExIsTENCE.--If́ we assume the objective reality of time and space, we must affirm that every thing which exists must cxist in time and space. But although existence necessarily implies time and space, it does not necessarily imply any measurable quantity or amount of time and space. A thing which exists, no matter what it may be, exists in the absolute and indivisible moment. Continued existence, of course, nccessarily implies an amount or measurable quantity of time, but not more existence abstractly considered.

The same does not, however, hold universally true in regard to space ; for whether the existence of a thing necessarily implies an extension or measurable quantity of space depends upon whether the thing possesses extension or not. For example, thoughts, feelings, emotions, \&c., and other menta] phenomena must exist in space, but do not require extension in space. Length, breadth, and thickness have no application to thought or to feelings. A mathematical point, for eximple, has position in space, but it does not occupy space.

Time and Space in relation to CHANGL.-Lapse of time is necessarily implied in the rery conception of change. Change is the passing from one state to another. The thing changed is first in the one state, and afterwards in the other. It cannot be in the two different states at the same moment.

Change does not, howerer, necessarily imply a quantitative amount of space as it does of time. Change implies succession of moments of time, the one following the other; but it does not necessarily imply a succession of positions in space. There can be no change without change of position in time; but there may be change without change of position in space. Change of thought, feelings, \&c. do not imply change of position in space. It may be true, or it may not, that montal change cannot take place without some change in our material organism (say, of the brain); but nevertheless the most rigid materialist will admit that change of thought itself does not imply change of position in space. Even in material objects change does not always imply change of position. A body may also be conceived to increase or decrease in density without any change in the position of its particles. Change of intensive quantity does not imply change of position in space.

Time and Space in velation to Transformation of energiy. - It will be perceived at once that lapse of time is necessirily implied in the very conception of transformation. Transfor- 
mation is a change from one form to another. There is first the one form, and then the other ; or, in other words, there is first the one form, and afterwards the other. The two are not simultaneous. It becomes the one by ceasing to be the other. Transformation without lapse of time is both inconceivable and a contradiction in terms.

If we leave out of consideration mental phenomena, we may affirm that transformation of energy without transposition in space is just as impossible as transformation of energy without transposition in time; only it is not so directly obvious in the one case as in the other. That transformation of energy necessarily implies change of position in space may be proved from general considerations alone, without reference to special eases.

Transformation of energy must consist either (1) of change of potential energy into kinetic, or (2) of kinetic energy into potential, or (3) of one form of kinetic energy into another form of kinetic energy, or (4) of one form of potential energy into another form of potential energy. There can be no other form of transformation beyond these ; for they comprehend the whole field of the possible. Now it is obvious that the first three forms of transformation imply change of position in space; for kinetic energy implies motion in some form or other, and motion implies change of position in space. The fourth and last form of transformation, viz. of one form of potential energy into another, were it possible, might, it is true; be conceived to take place without change of position; but there is evidently no such thing in nature, so far as is yet known, as one form of potential passing directly into another form. It is true that the potential energy of one form may be transformed into potential energy of another form; but it must, in the first instance, become kinetic before it assumes the second form. Static electricity, for example, may be transformed into magnetism, but not so directly. It must first assume the dynamic form before it can become magnetism. A magnet will magnetize a piece of soft iron, but before the iron assumes the magnetic or potential state a molecular change takes place. The first effect produced on the molecules of the iron is dynamic before they assume the static or magnetic state.

The conclusions to which we are led may be stated as follows :-

1st. Under every possible circumstance time and space are absolutely necessary as conditions to the transformation of energy from one form to another-the physical reason being that there can be no transformation without motion, and there can be no motion without time and space as conditions. 
2nd. In every possible case the amount of transformation is limited by the extent of the space condition available for the forces to act. We have seen that in the steam-engine, for example, the amount of energy in the form of heat that can be converted into mechanical work would be limited by the length of the cylinder, were it not that the stroke of the piston is reversed; for when the piston reached the end of the cylinder no further transformation of energy could take place. But the reversal of the motion of the piston affords an unlimited amount of space condition; consequently, so far as space is concerned, there is no limit to the amount of heat which may be converted into work in a steam-engine. The same thing is also true of the molecules of the steam itself. They could perform no work on the piston unless they had space to allow of motion. But as their motion, like that of the piston, is to and fro, there is no limit, so far as space is concerned, to the amount of energy which they may lose in work.

But gravity is a force which cannot act to and fro. The amount of energy which gravity can lose is limited by the distance between the gravitating bodies. When the space between them is traversed and the bodies are in contact, there is then no space condition, and further transformation of energy into work is impossible.

Energy of Position.-What is meant by energy of position must simply be that a body is in such a position that force may be converted into kinetic energy or energy of motion. It means that the force is in a position, so far as the space condition is concerned, of being transformed into kinetic energy. We do not speak of the piston of the stermengine being possessed of energy of position. We have no occasion to do so, simply because it is always in possession of energy of position. But we speak of the weight when raised and the bow when bent as possessing energy of position; for it is only when they are in this condition that force can be converted into motion. The generally received meaning, however, of these terms is not only erroneous and misleading, but bides a most important physical truth. For when it is said that a body possesses energy of position, it is not meant simply that the body, in regard to space, is in a position to allow of force being transformed into energy of motion; for it is not supposed that force is transformed at all, the only thing changing being simply the position of the body. When astone, dropped from a height, is being pulled to the ground by the force of gravity, it is supposed that there is no loss or consumption of gravity; the only thing lost which can possibly be regarded 


\section{Dr. J. Croll on the Transformation of Gracity.}

as the equivalent of the motion gained, is simply the distance between the stone and the ground. Consequently space is not only made a part of the potential energy, but the only part out of which the kinetic energy has been generatedspace or distance from the ground being the form in which the energy of the falling stone previously existed.

Gravity a Space-pervading Force.- The fact that gravity increases inversely as the square of the distance may be regarded as evidence of the truth of the views advocated by Faraday*, Waterston $\dagger$, and others, that it is a force pervading space external to bodies, and that on the mutual approach of the bodies this force is not increased as is generally supposed, but the bodies merely pass into a place where the force exists with greater intensity; for in such a case the intensity of the force, in the space external to any body, is inversely as the square of the distance from the centre of convergence of these lines of force. When a stone, projected upwards, recedes from the earth, its vis viva is transferred to space, and exists there as gravity. When the stone approaches the earth, the force existing in space is transferred back to the body and reappears as vis viva.

"The integral of gravitation," says Mr. Waterston, "is a function of space. . . . Each element of radial distance has associated with it a fixed element of mechanical force, to be given or taken from all bodies traversing it."

Gravity supposed to be an Impact or a Pressure.-It is now generally admitted that the conception of attraction does not represent the modus operandi of gravitation, because attraction implies action at a distance, or, in other words, that a thing acts where it is not, which is just as impossible as that a thing can act when it is not. Gravity, in all probability, is of the nature of an impact or a pressure. Some of our most eminent physicists state that the force of gravity must either result from impact of ultramundane corpuscles, in some respects analogous to that of the particles of a gas (which has been found to be capable of accounting for gaseous pressure), or it must result from difference of pressure in a substance continuously filling space, except where matter displaces it.

That gravity is a force of the nature of pressure is, I think, beyond all doubt; but that this pressure results from the impact of corpuscles or from difference of pressure in a substance filling space is purely hypothetical. Why not assume it to

* Phil. Mag, April 1857: Proceedings of the Royal Institution for 1855.

$\dagger$ Phil. Mag. [IV.] vol. xv. 
be a force without calling in the aid of corpuseles or a medium filling space?

On a former occasion * I endeavoured to show at considerable length that our inability to conceive how force can exist without a material medium has its foundation in a metaphysical misconception.

Force cannot be conceived to exist of itself ; for it is not a substance, but must be either the property of a substance or the effect of a cause. But there is no à priori necessity for forming any conception of the nature of the substance of which it is the property, or of the cause of which it is the effect. This is not all. To suppose that the substance of which the force is the property should be some material tangible thing such as a corpuscle, is to suppose that we must not only know the nature of the substance, but should also be able to form a sensuous representation of it. Here is a double error ; for it is only the properties that are cognizable through the senses; or, in other words, we know substance only through its properties, or in the way that it manifests itself. Every thing in the corpuscle, for example, which is manifested to the eye, to the ear, or to the touch, is just as much a property or an effect as is force itself. All that the intellect demands is that the force of gravity be the force of something; but what that something is other than a something manifesting itself as force we have no necessity for knowing. It does not follow that this something should have the additional properties of hardness, length, breadth, and thickness, \&c., so that it may be designated a corpuscle, a material particle, or an elastic medium \&c.

Grarity as a Retarding Cause.-I shall now briefly refer to a curious consequence which appears to follow from the impact theory, independently of any considerations which hare been here advanced regarding the transformation of gravity. If gravity results from corpuscular impact, it follows, although the contrary seems to have been generally assumed, that the force of impact will be greater when a body is moving in opposition to the corpuscles than when moring in the same direction, unless we assume, what would be absurd, that they move with infinite velocity. The collision in the case of two trains meeting each other is more severe than in the case of the one overtaking the other.

It therefore follows that, even though the atmosphere offered no resistance, a body projected rertically upwards would not return to the earth with absolutely the same velocity as it left. A stone, for example, projected apwards against an cxcessirely

* Philosophy of Theism. Ward and Co.: 1857. 
heavy shower of hailstones, will, irrespective of resistance from the air, return to the ground with a certain loss of motion, because the force of impact of the hailstones will be greater on the stone when ascending than when descending: Now if we substitute gravific corpuscles for hailstones we shall have the same result, though, of course, to a far less extent, owing to the enormous velocity of the gravific corpuscles compared with that of the hailstones. But unless these corpuscles move with infinite velocity, the force of impact cannot be absolutely as great on the descending as on the ascending stone; and if so it cannot return to the earth with absolutely the same velocity as it left. There must be a loss of motion, however small that loss may be.

In all probability this is a point too nice ever to be determined by experiment. This loss of motion might, however, if real, be detected in the ease of comets or planets with very eccentric orbits. The tendency of this cause would be to produce a shortening of the major axis of the orbit of the planet or comet, and of course a corresponding decrease in the period of revolution.

If gravitation were an impact, no planet nor comet could move everlastingly in an elliptic orbit. But the mutual disturbing forces of the planets will always maintain them in elliptio orbits; and it would therefore seem to follow that gravity alone, without any resisting medium, would ultimately bring the planets to the sun.

The same results will follow on the supposition that gravity is caused by unequal pressure in a substance continuously filling space.

XXXIII. On the Dynamical Signification of the Quantitics occurring in the Mechanical Theory of Heat. By C. SzILY, of Buda-Pesth*.

$0^{\mathrm{NE}}$ of the most important physical fundamental notions is that of temperature; all the quantities of the theory of heat, nay, every physical quantity stands in relation to this ; and yet there is perhaps not a single notion in the entire range of physics which has been so indefinite and obscure. Both the rational definition and the measurement of temperature have for their sole basis the frequently discussed but not

* Translated from a German version, communicated by the Author, of a memoir which appeared in the Hungarian Müegyetemi Lapok, vol. i p. 165. On perusing the proof sheet of the English translation, the author has made a few modifications, and inserted the results of some of his new investigations on the subject. 